\title{
Effects of a 12-Week Lifestyle Intervention on Novel Biomarkers for Type 2 Diabetes (T2D) in Obese Latino Youth
}

\author{
Jared Rosenberg', Armando Peña², \\ Gabriel Q. Shaibi2 , \& Joon Young Kim¹ \\ 1 Syracuse University, New York, USA \\ 2 Center for Health Promotion and Disease Prevention, Arizona State University, USA
}

\begin{abstract}
In obese non-diabetic youth, glucose response curve (GRC) and 1-hr glucose concentration during an oral glucose tolerance test (OGTT) represent novel biomarkers for T2D risk. Obese youth with monophasic- vs. biphasic-GRC and 1-hr glucose concentration of $\geq 155$ (Above 155) vs. $<155 \mathrm{mg} / \mathrm{dL}$ (Below155) are at increased risk for T2D. However, to date, it is unknown whether these OGT-derived phenotypes can be improved in response to any interventions, thereby hindering their practical use as indicators of intervention effectiveness. The purpose of this was to investigate the effects of lifestyle intervention on OGTT-GRC and 1-hr glucose concentration in obese Latino youth at increased risk for T2D.
\end{abstract}

Keywords: Type 2 diabetes risk, glucose response curve, 1-hr glucose concentration, lifestyle intervention

\begin{tabular}{lr}
\hline Article History & Corresponding Author \\
Received 16 September 2020 & Joon Young Kim \\
Accepted 1 October 2020 & jkim 29 syr.edu \\
Published 31 January 2021 & Department of Exercise Science \\
Available online 19 February 2021 & The David B. Falk College of Sport and Human Dynamics \\
https://doi.org/10.47544/johsk.2021.2.1.11 & Syracuse University, USA \\
\hline
\end{tabular}

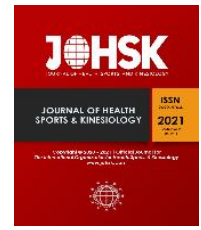

\section{Methods}

Sixteen obese Latino youth (age 15.6 \pm 0.9 years; 7M/9F; body mass index \%tile=98.3 \pm 1.2 ) completed a 12-week lifestyle intervention that included weekly nutrition education and 180 minutes of moderate-vigorous exercise per week. All participants completed a 2-hr OGTT before and after the lifestyle intervention to assess changes in OGTTGRC \& 1-hr glucose as well as other pathophysiological risk factors including insulin sensitivity index, insulinogenic index, and oral disposition index (oDI). Chi-square and paired $t$ test were used to compare changes in response to the intervention.

\section{Journal of Health, Sports, \& Kinesiology | ISSN 2692-9864 | www.johsk.com}




\section{Results}

At baseline, the prevalence of biphasic-GRC and Below 155 was $12.5 \%$ and $43.8 \%$, respectively. After the 12 -week intervention, OGTT-derived phenotypes were improved, exhibiting significant increases in the prevalence of biphasic-GRC (37.5\%, P=0.05) and Below 155 (75\%, P=0.042). Together with improvement on GRC and 1-hr glucose, ODI was enhanced (Pre: $7.36 \pm 6.20$ vs. Post: $8.16 \pm 5.13, P<0.05)$, despite no improvement in insulin sensitivity index and insulinogenic index.

\section{Discussion}

A 12-week lifestyle intervention is efficacious in improving glucose response curve and 1-hr glucose concentration during an OGTT in conjunction with B-cell improvement in obese Latino youth. Our data further suggest that these emerging T2D risk biomarkers have prospective utility in terms of assessing change following interventions/therapeutic trials.

\section{Journal of Health, Sports, \& Kinesiology | ISSN 2692-9864 | www.johsk.com}

